\title{
AGROECOSISTEMAS INTEGRALMENTE SANOS FRENTE A FORMAS AGROPRODUCTIVAS
}

\section{Agro ecosystem fully healthy food to ways agro production}

\author{
CARLOS ARTURO FRANCO ${ }^{1}$ \\ 1Universidad Tecnológica de Pereira
}

E-mail: carlosarturo.franco@gmail.com

Recibido: 26 de Agosto de 2010

Aceptado: 11 de Marzo de 2011

\section{Resumen}

Este artículo hace una primera aproximación a los aportes desde la agroecología a un agro ecosistema integralmente sano, y cuestiona la bondad de las agriculturas de precisión y convencional en este sentido. Para el autor, un agro ecosistema integralmente sano es aquel simultáneamente saludable para los ecosistemas que lo sustentan, y para todos los seres e instituciones involucrados en la cadena de agregación de valor hasta el consumidor final y la cadena de retorno de residuos a los resumideros ecos sistémicos. Saludable en todas las dimensiones de la vida: territorial, ecológica, bio-psico-neuro-emocional, espiritual, cultural, tecnológica, social, política y económica.

Palabras claves: Adaptación, Regeneración, Agroecosistema.

\section{Abstract}

This article is a first approach to the contributions from agro ecology agro ecosystem to fully healthy, and questions the goodness of precision agriculture and conventional in this regard. For the author, a fully healthy agro ecosystem simultaneously is that healthy ecosystems that sustain it, and for all people and institutions involved in the chain of added value to the consumer and the chain of return of waste to drains ecosystem . Healthy in all aspects of life: territorial, environmental, biopsycho-neuro-emotional, spiritual, cultural, technological, social, political and economic.

Keywords: Adaptation, Regeneration, Agroecosystem. 


\section{INTRODUCCIÓN}

Sobre los $13.971 \mathrm{~km} 2$ de suelo y agua del Eje Cafetero se realizan demandas contradictorias de bienes y servicios ambientales. De un lado están las expectativas y necesidades de adaptación al cambio climático, producción de alimentos en suficiente cantidad y calidad para una población local de 2.453.051 habitantes en 2010, que se proyecta a 2.557.606 hacia 2025 (DANE 2010) y terrenos para infraestructura urbana, comercial, industrial y de servicios. Del otro lado las políticas globalizadoras instan a producir para un mercado externo, insumos para biocombustibles, extracción minera a cielo abierto $y$, paisajes para ecoturismo.

Para responder a las necesidades de alimentos y adaptación al cambio climático en el Eje Cafetero los agroempresarios eligen entre dos grupos de formas productivas. En el grupo moderno-industrial se ubican la agricultura convencional y la transgénica. Siguen las reglas del mercado global, son dependientes del uso intensivo de capital financiero para sostener su capacidad de compra de mano de obra, maquinaria, insumos de síntesis química, semillas genéticamente modificadas y consumo de grandes cantidades de energía. Han evidenciado insostenibilidad en el tiempo y el espacio debido a su generación de pobreza, hambre, conflictos sociales y degradación de los ecosistemas que los sustentan.

En el grupo alternativo se encuentran: primero, la agroecología, mayormente adoptada por pequeños propietarios de predios que haciendo uso de su soberanía alimentaria producen bienes y servicios ambientales siguiendo procesos restauradores del ecosistema, y sanos para los actores involucrados el agro ecosistema.

Pese a sus bondades, en el Eje Cafetero las formas agroecológicas de producción de alimentos son incipientes en volumen de producción, superficie de tierra productiva, cantidad y variedad de alimentos ofertados. Para su expansión deben competir con el grupo dominante de agroproducción y superar barreras financieras, jurídicas, políticas, sociales, culturales y económicas.

Segundo, la agricultura de precisión, cercana a la agroecología en el propósito de restaurar y conservar los ecosistemas; desarrolla metodologías útiles para grandes y medianos empresarios del campo ligados a la agricultura convencional, moderna, industrial; poseedores de las mejores tierras y la mayor superficie. No es accesible por costos a pequeños propietarios con baja capacidad de inversión, los que son mayoría en Colombia, ubicados en tierras vulnerables y pobres.

Para el autor, un agro ecosistema integralmente sano es aquel simultáneamente saludable para los ecosistemas que lo sustentan, y para todos los seres e instituciones involucrados en la cadena de agregación de valor hasta el consumidor final y la cadena de retorno de residuos a los resumideros ecos sistémicos. Saludable en las dimensiones de la realidad: espiritual, ecológica, territorial, social, cultural, tecnológica, política, económica y bio-psico-neuro-emocional.

El autor establece una comparación entre los aportes desde las agriculturas convencional, de precisión y agroecológica, a un agro ecosistema integralmente sano.

\section{DESCRIPCIÓN}

El desarrollo y crecimiento socioeconómico en el mundo responde a una cultura que se fue centrando y reduciendo a lo meramente económico, en desmedro de otras dimensiones del ser humano, de sus creaciones sociales y de su entorno natural. Así se ha desembocado en crisis ambientales, sociales, económicas, que ineludiblemente llevan a dilemas entre crecer indefinidamente en un medio natural finito, $\mathrm{o}$ prever límites al crecimiento económico (Mejía 2008, Martínez 2009, Max-Neef et al. 2009).

Más de 6000 millones de personas dependen del alimento que se produce en el $11 \%$ de la superficie terrestre. En los últimos 20 años se ha registrado perdida de la capacidad productiva de los suelos: $40 \%$ en áfrica, $38 \%$ en Asia, 32\% en América Latina, $18 \%$ en USA, $12 \%$ en Europa (Asamblea General de las Naciones Unidas 1997). Los suelos tropicales son más frágiles que muchos otros, pero es en sus ecosistemas donde la humanidad tiene puestas las esperanzas para el abastecimiento mundial de alimentos en las próximas décadas (Zúñiga et al. 2009).

El sistema alimentario convencional no ha respondido de forma efectiva a las necesidades de la población mundial. Hoy, más de mil millones de personas padecen hambre (FAO 2006). En el Eje Cafetero, en 12 corregimientos y 19 comunas del municipio de Pereira, se encontró malnutrición global por 
déficit en $36.9 \%$ de los menores de cinco años, 30.1\% de los menores de 10 años y $20.9 \%$ de adolescentes de 10 a 17 años (Tabima 2008) entre una población de 451.645 habitantes en 2008).

En 1997 la Asamblea General de la ONU reconoció que "proporcionar seguridad alimentaria sustentable (y vivienda adecuada) constituye el desafío más grande que enfrenta la humanidad" (FAO 1996). La Seguridad Alimentaria y Nutricional (SAN) según la FAO es: "el derecho de todas las personas a tener en todo momento acceso físico y económico a suficientes alimentos inocuos y nutritivos para satisfacer sus necesidades alimenticias y sus preferencias, a fin de llevar una vida activa y sana" (FAO 2001).

Para el gobierno colombiano SAN es: "la disponibilidad suficiente y estable de alimentos, el acceso y el consumo oportuno y permanente de los mismos en cantidad, calidad e inocuidad por parte de todas las personas, bajo condiciones que permitan su adecuada utilización biológica, para llevar una vida saludable y activa" (Consejo Nacional de Política Económica y Social de Colombia 2005).

\section{La Agroproducción Convencional}

La primera Revolución Verde utilizó un paquete tecnológico con prácticas agrícolas basadas en el empleo masivo de fertilizantes de síntesis química, el uso de agro tóxicos (herbicidas, insecticidas, fungicidas, nematicídas, etcétera), el sobre-laboreo, las quemas, la compactación por mecanización excesiva, el riego con aguas inapropiadas, la pérdida de las variables hidráulicas y la contaminación; así generó problemas de malezas, plagas, consumo creciente de fertilizantes, fungicidas, herbicidas, insecticidas, etc., que concluyeron en suelos degradados y disminución del rendimiento en el largo plazo (Altieri \& Anderson 1986).

Entre 1960 y 1980, la aplicación de fertilizantes era mínima en relación con las producciones obtenidas (Martínez 2009), pero la forma de agro producción convencional, totalmente dependiente de fertilizantes, incorporó nitrógeno a base de urea, una materia prima con precios atados a los del petróleo con una tendencia de aumento creciente (Altieri 2002). Este énfasis en la adquisición de insumos químicos, de origen ajeno al predio agrícola, elevó los costos y menguó las cosechas, aumentando la desigualdad (Martínez 2009, Puche 2010).
En 2010 se requieren mayores cantidades de insumos para obtener menores producciones a las de 1980. Para los pequeños y medianos campesinos que constituyen la mayoría de los productores en el mundo y sobre los cuales recae la soberanía alimentaria y territorial es cada vez menos posible recuperar los costos de su producción por medio de los precios de mercado.

Estas circunstancias aunadas al crecimiento de la población mundial, la demanda de biocombustibles, la minería a cielo abierto, la dispersión urbana, han dado inicio a una crisis alimentaria, ambiental, social con tendencia a acentuarse en el medio y largo plazo.

\section{La agricultura moderna industrial - biotecnológica}

La agricultura moderna ha resuelto problemas a muy alto costo; en efecto, en el mundo se emplean más de 2000 millones de $\mathrm{kg}$ de pesticidas, que producen problemas de contaminación ambiental y afectan la salud de fauna, flora, agricultores y consumidores.

La primera revolución verde, dirigida por el sector público, tenía como objetivo manifiesto proporcionar más comida, y como objetivo oculto vender más productos químicos, lo que expandió la dependencia del uso de químicos a cambio de insumos orgánicos domésticos y conocimiento tradicional de los agricultores.

La segunda revolución verde plantea que la sobrepoblación, la baja productividad agrícola la escasez de alimentos son la causa del hambre, y que la solución es la expansión de semillas transgénicas y la dependencia total de los agricultores de la multinacional Monsanto. Estas relaciones entre la producción y el consumo de alimentos a nivel mundial, tratan de invisibilizar la distribución desigual de oportunidades y las disparidades existentes dentro de cada país (Altieri \& Anderson 1986).

Esta nueva Revolución opera un modelo agroindustrial fundado en el uso intensivo de fertilizantes, plaguicidas, semillas modificadas genéticamente (Mejía 2008) y políticas comerciales que faciliten la uniformidad y estandarización de tecnologías para aplicar sistemas mecanizados que requieren insumos en gran escala (Altieri 2002).

El objetivo de esta revolución es aumentar los beneficios de las multinacionales con Monsanto como líder que busca 
imponer en el mundo la ley de las patentes. Una vez impuesta la norma del derecho de propiedad de los granos modificados genéticamente se podría cobrar royalties ${ }^{1}$; por cada grano sembrado y para cada campo cultivado. El gobierno estadounidense presiona a cada gobierno para que aplique el acuerdo relativo - a los aspectos de la propiedad intelectual que afectan al comercio (ADPIC) de la Organización Mundial de Comercio (OMC) (Robin 2008).

La introducción indiscriminada de semillas modificadas genéticamente pone a los agroecosistemas en peligro, puede reducir la diversidad genética de cultivos alimenticios, y elevar el riesgo y la inseguridad agroalimentaria en varias zonas (Puche 2010).

Los cultivos transgénicos dependen del glifosato, realizan un exudado continuo de toxina $\mathrm{Bt}^{2}$ por el maíz y provocan una gran erosión genética (por ejemplo el $80 \%$ de la colza ya está contaminada) (Halweil et al. 2010), por esta razón la agricultura biotecnológica no sería una forma sustentable de garantizar seguridad alimentaria (Altieri 2002). Además, el dumping de alimentos excedentarios de la agricultura industrial que practica Estados Unidos es uno de los factores que contribuye a reducir la productividad de los países empobrecidos, lo que hace inviable este método para luchar contra el hambre (Franco 2004, Suarez 2007, Roa 2009). La concentración anual de dióxido de carbono $(\mathrm{CO} 2)$ en la atmósfera presenta una tendencia promedio creciente desde (Mauna Loa Observatory 2010) (Tabla 1, Figura 1).

Este cambio podría indicar que la Tierra está perdiendo su capacidad natural para absorber millones de toneladas de $\mathrm{CO} 2$ al año, síntoma del avance hacia niveles peligrosos del calentamiento global (Revista electrónica La Vanguardia) frente a lo que la agricultura industrial actúa como acelerador (Altieri 2004).

\section{La Agricultura de Precisión: una visión analítica -sintética del suelo.}

\footnotetext{
${ }^{1}$ Royalty: cantidad que ha de pagarse por el uso de una propiedad industrial, patente, marca, etc., registrada a nombre de otro. http://www.finanzasdigital.com/servicios_glosario.php

${ }^{2}$ El Bt (Bacillus thuringiensis) es una bacteria que se utiliza en agricultura ecológica y también en ingeniería genética como genes incorporados al organismo receptor, que le permiten producir una sustancia insecticida o tóxica.
}

Un aspecto subyacente a la agricultura industrial es su concepción mecanicista de la naturaleza, reduciendo la explicación de todas sus propiedades a sus partes componentes: química, física, biológica y fitosanitaria; obviando un enfoque holístico o sintético, con énfasis en el todo (Zúñiga et al. 2009).

Para los creadores de la Agricultura de Precisión (AP) es complementaria la visión analítica con la visión sintética, por lo que en el estudio de la producción agrícola integran el conocimiento fraccionado (física, química y biología de suelos), con el conocimiento holístico de la dinámica del suelo buscando el manejo sostenible de los recursos naturales y la producción armónica de los mismos (Zúñiga et al. 2001).

La AP percibe el suelo como un sistema emergente ordenado según criterios de auto-organización y resiliencia por la interacción de diversas fases: una lineal, donde son válidos los análisis euclidianos; otra hecha de sistemas no lineales que involucra estructuras fractales y sintéticas; y una fase de complejidad propiamente que involucra procesos de caos, irreversibles y de no linealidad (Zúñiga et al. 2009).

Tabla 1. Tendencia anual de CO2 en la atmosfera. Tomado de (Mauna Loa Observatory 2010).

\begin{tabular}{lllllllll}
\hline \multicolumn{1}{c}{$\begin{array}{c}\text { año } \\
\text { ppm/año }\end{array}$} & \multicolumn{2}{c}{ año } \\
ppm/año & \multicolumn{3}{c}{$\begin{array}{c}\text { año } \\
\text { ppm/año }\end{array}$} & \multicolumn{3}{c}{$\begin{array}{c}\text { año } \\
\text { ppm/año }\end{array}$} \\
\hline 1959 & 0.95 & 1971 & 0.82 & 1984 & 1.37 & 1997 & 1.96 \\
1960 & 0.51 & 1972 & 1.76 & 1985 & 1.24 & 1998 & 2.93 \\
1961 & 0.95 & 1973 & 1.18 & 1986 & 1.51 & 1999 & 0.94 \\
1962 & 0.69 & 1974 & 0.78 & 1987 & 2.33 & 2000 & 1.74 \\
1963 & 0.73 & 1975 & 1.10 & 1988 & 2.09 & 2001 & 1.59 \\
1964 & 0.29 & 1976 & 0.92 & 1989 & 1.27 & 2002 & 2.56 \\
1965 & 0.98 & 1977 & 2.09 & 1990 & 1.31 & 2003 & 2.29 \\
1966 & 1.23 & 1978 & 1.31 & 1991 & 1.02 & 2004 & 1.55 \\
1967 & 0.75 & 1979 & 1.68 & 1992 & 0.43 & 2005 & 2.55 \\
1968 & 1.02 & 1980 & 1.80 & 1993 & 1.35 & 2006 & 1.72 \\
1969 & 1.34 & 1981 & 1.43 & 1994 & 1.90 & 2007 & 2.17 \\
1970 & 1.02 & 1982 & 0.72 & 1995 & 1.98 & 2008 & 1.66 \\
& & 1983 & 2.16 & 1996 & 1.19 & 2009 & 1.98 \\
\hline
\end{tabular}

Al comparar un ecosistema de bosque sin intervenir y un ecosistema intervenido con labranza intensiva, se encuentra que en el ecosistema de bosque sin intervenir: 
(1) La densidad aparente y la conductividad térmica del suelo es menor que en el ecosistema intervenido. (2) La energía libre de Gibbs es mayor que en el ecosistema intervenido. (3) La Reserva Energética del Suelo o el Potencial Productivo del Suelo (PPS) es mayor que en el ecosistema intervenido lo cual indica una pérdida de energía del suelo cuando se aplica una labranza intensiva (López 2006).

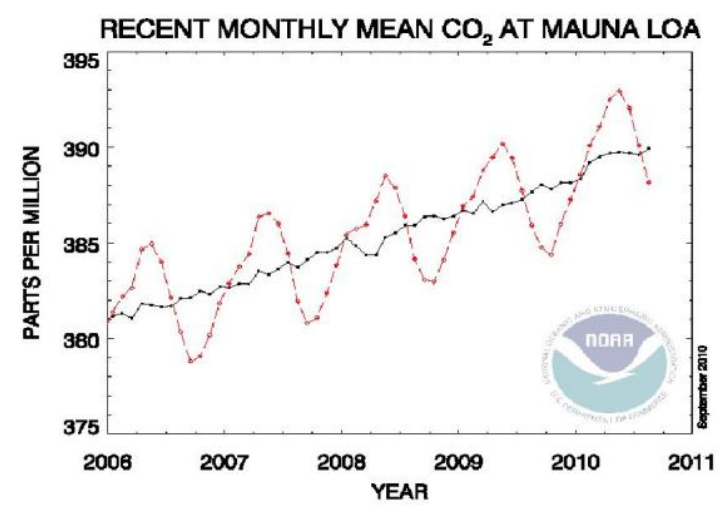

Figura 1. Tendencia anual de $\mathrm{CO}_{2}$ en la atmosfera. Tomado de Mauna Loa Observatory (2010).

Atendiendo al anterior hallazgo, la AP formula una concepción de las ciencias del suelo que profundiza en la relación entre las variables tradicionalmente medidas que brindan información sobre el estado de la materia en el suelo (masa), y aquellas que ofrecen información sobre su estado energético (conductividad térmica, resistividad eléctrica, potencial productivo) (Zúñiga et al.2009).

El Potencial Productivo del Suelo (PPS) es un indicador representativo de su estado de bienestar. Su cálculo es función de parámetros analíticos a los que da importancia uniforme, y sintéticos. $\mathrm{Su}$ determinación es necesaria para realizar un análisis sintético del suelo (Zúñiga, et al. (2001), e inferir con mayor precisión sobre la sostenibilidad, la eficiencia y la productividad del manejo de suelos en el mediano y largo plazo (López 2006), así como planificar una fertilización diferencial del cultivo en estudio, lo que reduce el uso indiscriminado de insumos, la contaminación y los costos de producción (Orellana 1972, Duarte 2006, Zúñiga et al. 2009).

Las propiedades físicas del suelo: humedad, densidad aparente, macroporos, microporos, determinan la rigidez y la fuerza de sostenimiento, la facilidad para la penetración de las raíces, la aireación, la capacidad de drenaje y de almacenamiento de agua, la plasticidad, y la retención de nutrientes; y suplementan las necesidades de oxígeno, carbono e hidrógeno a las plantas y la solubilidad de muchos nutrientes (Rucks et al. 2004).

Una propiedad biológica del suelo indispensable para su buena fertilidad y una buena producción del cultivo es la actividad de los microbios sobre el suelo. Estos descomponen la materia orgánica para dar humus, reúnen partículas del suelo para dar una mayor estructura, protegen las raíces de diferentes enfermedades y parásitos, retienen el nitrógeno y otros nutrientes, producen hormonas que ayudan a las plantas a crecer y pueden convertir los contaminantes que encuentran en el suelo (García 2010).

Tabla 2. Resumen de propiedades a cuantificar. Tomado de Zúñiga et al. (2009). Alternativas en el manejo sostenible de los suelos: un enfoque analítico y sintético.

\begin{tabular}{ll}
\hline Propiedades sintéticas & Unidades \\
\hline Conductividad térmica & $\mathrm{Cal} / \mathrm{cm} . \mathrm{s} .{ }^{\circ} \mathrm{C}$ \\
Resistividad eléctrica & $\mathrm{Ohm} . \mathrm{m}$ \\
Impedancia & $\mathrm{Q}$ \\
\hline Propiedades analíticas & Unidades \\
\hline Materia orgánica & $\%$ \\
Densidad aparente & $\mathrm{g} / \mathrm{cm}^{3}$ \\
Macroporos & $\%$ \\
Microporos & $\%$ \\
Espacio poroso total & $\%$ \\
Actividad microbiana & $(\mathrm{igC}-\mathrm{CO} / \mathrm{gSS}$ \\
Porcentaje de humedad & $\%$ \\
Porcentaje de arena & $\%$ \\
Potencial de hidrógeno & $\mathrm{\% scala} \mathrm{de} \mathrm{pH}$ \\
Saturación de bases & $\%$ \\
CIC & $\mathrm{cmol} / \mathrm{kg}$ \\
Conductividad eléctrica & $\mathrm{dS} / \mathrm{m}$ \\
(Ca+Mg)/K & - \\
Fósforo & $\mathrm{ppm}$ \\
Potasio & $\mathrm{cmol} / \mathrm{kg}$ \\
Elementos menores & $\mathrm{mg} / \mathrm{kg}$ \\
\hline
\end{tabular}

La resistividad eléctrica ${ }^{3}$ es una medida de la dificultad que la corriente eléctrica encuentra a su paso por un material determinado, donde los registros responden a una

\footnotetext{
${ }^{3}$ Patente de Invención Europea \# 9600407. Zúñiga. O, Gascó, J. M. (1999). "Electro sonda para medir el contenido de humedad del suelo y la compactación del mismo, método de medida correspondiente y utilización de dicha electro sonda".
} 
combinación entre la humedad, la concentración de iones solubles y el tipo físico del suelo, siendo regida por el número y la movilidad de su carga libre (es decir, los iones solubles) (López 2006).

\section{La Agroecología}

Una forma alternativa a la agricultura industrializada y de precisión, es la campesina. Según Ángel Palerm, Víctor M. Toledo, Stephen R. Gliessman y Miguel A. Altieri, la Agroecología es un cuerpo teórico que contiene las bases científicas para acometer la lucha por la seguridad alimentaria y nutricional.

Los programas de desarrollo rural promovidos «desde abajo» sugieren cuatro requisitos básicos para una estrategia ecológica: (1) Uso de tecnologías adaptables basadas en prácticas tradicionales, tecnologías autóctonas y germoplasma criollo; (2) Énfasis en el empleo de tecnologías fácilmente comunicables de un agricultor a otro, y por lo tanto que utilicen experimentación en pequeña escala y demuestren un efecto oportuno; (3) Compromiso de los campesinos en el diseño, elaboración, manejo y evaluación del programa, y empleo de personal local en calidad de promotores; (4) Utilización de métodos pedagógicos de demostración sobre la base del principio de aprendizaje mediante la práctica (Galindo 2006).

La producción agroecológica apuesta a un modo alimentario, humano y sostenible basado en principios de "producción y consumo responsable", asociatividad para la comercialización directa y la distribución en circuitos cortos, gestión cooperativa del conocimiento entre productores $y$ consumidores, producción y consumo de alimentos de temporada, y dedicación de recursos sociales para hacer dignos la vida y el trabajo en el campo (Restrepo 2005).

Los campesinos que adoptan y adaptan las prácticas citadas gozan de mayor autosuficiencia alimentaria y se consolidan más a nivel comunitario al colaborar recíprocamente en el trabajo y en otras actividades (Altieri. 2004). El modo agroecológico de apropiación del territorio activa factores que inducen procesos restauradores de los ciclos de vida naturales interferidos por la intervención dominante en las relaciones sociedad- naturaleza (Giddens 1990, Barry 2007)

Los movimientos sociales agroecológicos acogen para sus procesos productivos las prácticas de saber-hacer agroecológicas (López 2006). Sus formas de actuar responden a planteamientos del "pos desarrollo"4. Desde diversos campos culturales emergen muchos de los movimientos sociales actuales que, además de buscar satisfacer necesidades materiales, se plantean objetivos inasibles desde una perspectiva materialista: derechos culturales, identidades, economías alternas (no abocadas a la acumulación) (Escobar 2005).

La Agroecología ofrece alternativas rentables sin utilizar fertilizantes y fitosanitarios, como por ejemplo el maíz-soja en franjas, o colocar franjas de desmodium junto al maíz, para ahuyentar parásitos y estimular la presencia de insectos útiles (Galindo 2006).

Los agro ecosistemas de salud integral deben ser construidos por la comunidad trabajando simultáneamente sobre el control de todas las dimensiones de la realidad de la comunidad y el individuo: territorial, ecológica, cultural, social, política, económica, tecnológica, espiritual, y bio-psiconeuro-emocional.

El control sustentable se construye socialmente (Berger \& Luckmann 1968) desde adentro hacia afuera, con la participación responsable de todos y cada uno los miembros de la comunidad, haciendo uso del conocimiento y sabiduría local en interacción permanente con el entorno. Es un proceso de carácter endógeno de autodeterminación y autogestión que tiene como propósito la toma o recuperación del control de los procesos que determinan y afectan la comunidad (Toledo 2010). Así:

(1) El control territorial implica el deslinde de la superficie que le corresponde, el establecimiento de límites, el reconocimiento de su territorio por parte del Estado y de las comunidades (Toledo 2010).

(2) El control ecológico se logra con el uso restaurador o regenerador de los ecosistemas (flora, fauna, suelos, flujos de agua, etc.) que forman parte del territorio.

(3) El control cultural implica que la comunidad asuma el rescate y valoración de su patrimonio histórico y sus propios valores culturales, incluyendo la lengua, vestimentas, costumbres, conocimientos, creencias, hábitos, etc.

${ }^{4}$ Término utilizado desde un coloquio internacional celebrado en Ginebra en 1991. 
(4) El control social enfoca el incremento de la calidad de vida de los miembros de la comunidad. Atiende a necesidades de seguridad en alimentación, salud, educación, vivienda, sanidad, esparcimiento e información (Toledo 2010).

(5) El control económico es la regulación de los intercambios económicos que la comunidad y sus miembros realizan con el resto de la sociedad y con los mercados locales, regionales, nacionales e internacionales (Toledo 2010).

(6) El control político supone una capacidad de la comunidad para crear su propia organización (socio/productiva), promulgar o ratificar las normas, reglas y principios que rigen la vida política de la comunidad, y asegurar la participación de los miembros, la democracia comunitaria, la autonomía política y la ejecución del derecho consuetudinario (Toledo 2010). La igualdad abstracta de los ciudadanos debe transformarse en discriminación positiva, más realista, que reduce las desigualdades efectivas (Zin 2002)

(7) El control tecnológico cuida del desarrollo de tecnologías que mejoren el bienvivir de la comunidad en armonía con las prácticas y saberes ancestrales. Propende por las innovaciones como nuevas formas de hacer las cosas -ideas, pensamientos, inventos, acciones- que resuelven problemas de los diferentes aspectos de la vida o permiten que las personas aprovechen oportunidades (IDRC 2010)

(8) El control espiritual se funda en recuperar la conciencia de conexión de la naturaleza humana con los elementales tierra, agua, fuego y aire; y el conocimiento colectivo ancestral.

(9) El control bio-psico-neuro-emocional corresponde al plano individual; cada miembro de la comunidad se hace responsable de sus acciones, y establece relaciones entre su estilo de vida y su estado de salud actual y futuro.

Una construcción social del individuo, de la comunidad y de agro ecosistemas integralmente sanos, va de la mano de una producción histórica de la autonomía, que no es algo dado sino que debe lograrse mediante un ejercicio continuado de la planeación acción en círculo virtuoso repetitivo y auto mejorado con el tiempo. En el que todos los miembros de la comunidad se perciban actores y autores centrales. Este plan contiene propósitos y estrategias para cada una de las dimensiones.
Por ejemplo, para el control ecológico se formula un Plan de manejo ambiental, capaz de normar y regular las actividades agrícolas, pecuarias, forestales y pesqueras que la comunidad realiza. Dicho plan de manejo implica la elaboración de un diagnóstico, un inventario, y de ser posible, la elaboración de un Sistema de Información Geográfica, por medio del cual se evalúe la oferta ecológica de recursos del territorio de la comunidad.

La agroecología está llamada a ocupar un papel central en los planes de desarrollo de la comunidad. En general, después de 50 años de investigación, extensión y apoyo, se acepta que hay un rendimiento mayor del $10 \%$ en producción convencional frente a la ecológica. Es decir que con poco más de 20 años de investigación agroecológica, los resultados son atrayentes y lo serían más si esta ciencia se introdujera en la Universidades (Altieri 2004). Por esto la agroecología se reconoce hoy en día como la base para orientar la conversión de sistemas convencionales de producción (monocultivos dependientes de insumos agroquímicos) a sistemas más diversificados y autosuficientes (Restrepo 2010). En lo cultural se deberá crear mecanismos que garanticen el rescate cultural y la toma de conciencia por parte de los habitantes de la existencia de su propia cultura (orgullo étnico). En la dimensión económica se enfrentan de manera comunitaria los fenómenos económicos externos que afectan la vida productiva de la misma, tales como políticas de fijación de precios (por el mercado o por el Estado), políticas macroeconómicas, subsidios, impuestos, préstamos, etc. Ello supone atenuar los mecanismos que afectan, inhiben e incluso castigan la esfera productiva de la comunidad

Los proyectos agroecológicos promovidos por las ONGs han dado lugar a mayor producción, mejor distribución de ingresos o más empleo rural, pero se han difundido muy lentamente, ya que las situaciones de urgencia del campo han exigido más dedicación a la acción que a la investigación o la publicación de resultados (IMCA 2003).

\section{CONCLUSIONES}

La comunidad es el actor-autor del diseño y puesta en práctica de un Plan de desarrollo endógeno de agro ecosistemas alimentarios integralmente sanos, con una visión sintético-analítica de la interacción entre las dimensiones de los agros ecosistemas del territorio. 
La economía agroecológica puede aportar al diseño y construcción de un agro ecosistema integralmente sano, que sea simultáneamente saludable para los ecosistemas que lo sustentan, y para todos los seres e instituciones involucrados en la cadena de agregación de valor hasta el consumidor final y la cadena de retorno de residuos a los resumideros ecos sistémicos.

La agricultura de precisión debe avanzar en la adecuación de sus tecnologías al nivel adquisitivo de los pequeños y medianos agricultores. Las agriculturas, convencional e industrial son simplificadas, poco diversas, intensivas en insumos externos, poco accesibles a pequeños productores y opuestos a la vida de agro ecosistemas alimentarios integralmente sanos.

El modo de investigación mono disciplinar no estudia los agro ecosistemas en todas sus dimensiones. Un reto para la academia es aprender a coinvestigar con los campesinos para construir conocimiento ambiental, recuperar saberes y sistematizar nuevas prácticas para la expansión de agro ecosistemas alimentarios viables culturalmente y deseables socialmente.

\section{REFERENCIAS}

ALTIERI, M. A. 2002. Agro ecology: the science of natural resource management for poor farmers in marginal environments, Agriculture, Ecosystems \& Environment 93(13): $1-24$.

ALTIERI, M. A. 2004. La agroecología es una ciencia útil para la transición a la Agricultura Ecológica. España: Sociedad Española de Agricultura Ecológica (SEAE), 1.

ALTIERI, M.A. \& ANDERSON, M.K. 1986. An ecological basis for the development of alternative agricultural systems for small farmers in the Third World. American Journal of Alternative Agriculture 1: 30-38.

ASAMBLEA GENERAL DE LAS NACIONES UNIDAS. 1997. "Programa para la Ejecución Ulterior de la Agenda 21", Sesión Especial del 23 al 27 de junio de 1997, párrafo 62.

BARRY, J. 2007. Medio Ambiente y Teoría Social. La teoría social y el mundo no humano. Cap. 4 Canadá: siglo XX $2^{\mathrm{a}}$ ed.

BERGER, P \& LUCKMANN, T. 1968. La Construcción Social de la Realidad. Madrid: Amorrortu.
CONSEJO NACIONAL DE POLÍTICA ECONÓMICA Y SOCIAL DE COLOMBIA. 2005. Documento Conpes Social 113, p. 60

DANE, Departamento Nacional de Estadísticas. 2010. Censo poblacional.

DUARTE, L. 2006. Desarrollo de una metodología de evaluación del Potencial Productivo del Suelo como indicador de calidad en un cultivo de maíz (Zea mays L.)

Dekal B DK- 5005 en Cerrito Valle del Cauca. Trabajo de grado (Ingeniería Agrícola). Universidad del Valle, Universidad Nacional de Colombia.

ESCOBAR, A. 2005. El "postdesarrollo" como concepto y práctica social. En Daniel Mato (coord.), Políticas de economía, ambiente y sociedad en tiempos de globalización. Caracas: Facultad de Ciencias Económicas y Sociales, Universidad Central de Venezuela, pp. 17-31.

FAO, FOOD AND AGRICULTURE ORGANIZATION (FAO). 2009. El estado de la inseguridad alimentaria en el mundo 2008 Los precios elevados de los alimentos y la seguridad alimentaria: amenazas y oportunidades.

FAO, FOOD AND AGRICULTURE ORGANIZATION. 1996. Asamblea mundial.

FAO, FOOD AND AGRICULTURE ORGANIZATION. 2001. Documento borrador de la Misión de identificación del Apoyo al Programa de Desarrollo Rural para la Paz y el Programa Especial para la Seguridad Alimentaria- PESA, Oct., 2001. Disponible en: http:/ / books.google.com.co/

FRANCO, C A. 2004. Implicaciones del TLC en la agroindustria colombiana. Universidad de Bogotá Jorge Tadeo Lozano, Maestría en Mercadeo Agroindustrial.

GALINDO, P. 2006. Agroecología y consumo responsable. Teoría y práctica. Coord. Madrid: Kehaceres. 46 pp.

GARCÍA, R. 2010. La agroecología según los Genaros. Entrevista con Carlos A Franco. Agosto 7.

GIDDENS, A. 1990). Consecuencias de la modernidad. Madrid: Alianza.

HALWEIL, B. NIERENBERG, D. \& RUNYAN, C. 2010. La tragedia de las semillas transgénicas en la agricultura. 
Ambiente y Sostenibilidad 2011 (1): 39-47

Revista del Doctorado Interinstitucional en Ciencias Ambientales

ISSN: 2339-3122

Entrevista con Percy Schmeiser. Disponible en: http://www.percyschmeiser.com

IDRC, INTERNATIONAL DEVELOPMENT RESEARCH

CENTRE. 2010. Programa para el escalamiento de innovaciones rurales. Llamado para la presentación de propuestas de proyecto 02/2010.

IMCA, Instituto Mayor Campesino. 2003. Pastoral de la Tierra, Ed. $\mathrm{N}^{\circ}$. 47 julio 8 de 2003. Buga: http://www.elcatolicismo.com.co

LÓPEZ, L. 2006. Evaluación de la dinámica de la Reserva Energética del Suelo y su relación con la productividad en un cultivo de algodón en el municipio del Cerrito Valle del Cauca. Trabajo de grado (Ingeniería Agrícola). Universidad del Valle, Universidad Nacional de Colombia.

MARTÍNEZ, A. (2009). Economía ecológica. Disponible en: www.//icarialibros.blogspot.com/2009/02/decrecimiento sostenible-un-articulo-de.html.

MAUNA LOA OBSERVATORY. 2010. Earth System Research Laboratory. Hawai : U.S. Department of Commerce National Oceanic \& Atmospheric Administration NOAA Research. Disponible en: http://www.esrl.noaa.gov/gmd/obop/mlo/.

MAX-NEEF, M. ELIZALDE, A. HOPPENHAYN, M. 2009. Desarrollo a Escala Humana. Disponible en: http://www.neticoop.org.uy/IMG/pdf/DesEscalaHumana .pdf. p.3

MEJÍA, M. 2008. Agricultura y Espiritualidad. 2008. Disponible en: http:/ / bloglemu.blogspot.com/2008/11/agricultura-yespiritualidad.html

ORELLANA, E. 1972. Prospección geoeléctrica en corriente continua, Paraninfo, Biblioteca técnica Philips Madrid España, 23-25, 107-108 pp.

PUCHE, P. 2010. ¡Monsanto, mon Dieu! Revista El Observador 30-07-2010. Disponible en: http:/ / www.revistaelobservador.com/index.php?Option=co m_content\&task $=$ view\&id $=3958 \& \mathrm{I}$ temid $=64$

RESTREPO, D. 2010. ¿Qué significa la conversión ecológica? Blog Agroecología con el profesor Yarugyver. 21 de mayo de 2010.http://yarugyver.blogspot.com/2010/05/agroecologia. htm 1 .
RESTREPO. 2005. La Mierda de Vaca. Entrevista. www.lamierdadevaca .com/dpl/?q=node/16.

Revista Electrónica La Vanguardia. http://www .lavanguardia.es/lv24h/20080328/53448813544.html.

ROA, T. 2009. La cuestión agraria en Colombia. Disponible en: www.adital.org.br/site/noticia.asp?lang $=E S \& \operatorname{cod}=42437$

ROBIN, M.M. 2008 El mundo según Monsanto. De la dioxina a los OGM. Una multinacional que les desea lo mejor. Madrid: Península. Entrevista con Vandana Shiva (pp. 449 y ss) en diciembre de 2004.

RUCKS, L. et al. 2004. Propiedades Físicas del Suelo. Montevideo-Uruguay. Facultad de Agronomía. Universidad de la República, Dpto. de suelos y aguas.

SUAREZ, A. 2007. El modelo agrícola colombiano y los alimentos en la globalización. Ediciones Aurora, Bogotá. 227 pp.

TABIMA, D. et al.. 2008. Situación Nutricional. Menores de 18 años y Madres Gestantes. Municipio de Pereira 2008. Alcaldía de Pereira. pp. 27-92. Facultad de Ciencias de la Salud. Universidad Tecnológica de Pereira

TOLEDO, V. M. 2010. Principios etnoecológicos para el desarrollo sustentable de comunidades campesinas e indígenas. Red Latino Americana y Caribeña de Ecología Social.http://www.ambiental.net/biblioteca/ToledoEtnoeco logia. Htm

ZIN, J. 2002. Los actuales desafíos de la ecología política. Transversales science culture 1, nueva serie, primer trimestre. http://www.inisoc.org/zin65.

ZÚÑIGA, O. et al.. 2001. Estrategias de desarrollo y la concepción agroambiental: un enfoque analítico y sintético. Modulo I, Diplomado en Agricultura Orgánica, Universidad del Valle. 5-6 pp.

ZÚÑIGA, O. OSORIO, J. C. \& CUERO, R. 2009. Alternativas en el manejo sostenible de los suelos: un enfoque analítico y sintético. Grupo ILAMA. Laboratorio de Física Ambiental. Departamento de Física, Facultad de Ciencias Naturales y Exactas. Universidad del Valle, 1-11 pp. 